University of Nebraska - Lincoln

DigitalCommons@University of Nebraska - Lincoln

Ralph Skomski Publications

Research Papers in Physics and Astronomy

November 1994

\title{
Aligned two-phase magnets: Permanent magnetism of the future? (invited)
}

Ralph Skomski

University of Nebraska-Lincoln, rskomski2@unl.edu

Follow this and additional works at: https://digitalcommons.unl.edu/physicsskomski

Part of the Physics Commons

Skomski, Ralph, "Aligned two-phase magnets: Permanent magnetism of the future? (invited)" (1994). Ralph Skomski Publications. 15.

https://digitalcommons.unl.edu/physicsskomski/15

This Article is brought to you for free and open access by the Research Papers in Physics and Astronomy at DigitalCommons@University of Nebraska - Lincoln. It has been accepted for inclusion in Ralph Skomski Publications by an authorized administrator of DigitalCommons@University of Nebraska - Lincoln. 


\title{
Aligned two-phase magnets: Permanent magnetism of the future? (invited)
}

\author{
R. Skomski \\ Department of Pure and Applied Physics, Trinity College, Dublin 2, Ireland
}

\begin{abstract}
Micromagnetic calculations are used to investigate coercivity and energy products of magnets consisting of an aligned hard-magnetic skeleton phase and a soft-magnetic phase with high saturation magnetization. Compared to the present-day theoretical limit of $516 \mathrm{~kJ} / \mathrm{m}^{3}$ for single-phase $\mathrm{Nd}_{2} \mathrm{Fe}_{14} \mathrm{~B}$, the energy product in suitable nanostructured $\mathrm{Sm}_{2} \mathrm{Fe}_{17} \mathrm{~N}_{3} / \mathrm{Fe}_{65} \mathrm{Co}_{35}$ composites is predicted to be as high as $1090 \mathrm{~kJ} / \mathrm{m}^{3}$. The influence of the skeleton's texture and shape is discussed, and aligned nanocrystalline two-phase magnets are compared with remanence-enhanced isotropic magnets. In particular, it is shown how the nucleation-based analytical approach breaks down in the isotropic limit. Finally, we outline conceivable processing methods and discuss potential applications of "megajoule" magnets.
\end{abstract}

\section{INTRODUCTION}

A key figure of merit of permanent magnetic materials is the energy product $(B H)_{\max }$, which describes the ability to store magnetostatic energy. Energy product increases with coercivity $H_{c}$ and remanence $M_{r}$ but can never exceed the value $\mu_{0} M_{r}^{2} / 4$ corresponding to an ideal rectangular hysteresis loop. Since $M_{r} \leqslant M_{0}$, the spontaneous magnetization $M_{0}$ yiclds an "intrinsic" limit $(B H)_{\max } \leqslant \mu_{0} M_{0}^{2} / 4$, but if magnetization were the only consideration then $\alpha$ iron with $\mu_{0} M_{0}=2.15 \mathrm{~T}$ would be used for permanent magnets with energy products as high as $920 \mathrm{~kJ} / \mathrm{m}^{3}$. In fact, the coercivity of bcc iron is so low that energy products of iron magnets are only of order $1 \mathrm{~kJ} / \mathrm{m}^{3}$, and in the past it was necessary to resort to cumbersome bar and horseshoe shapes to avoid spontaneous demagnetization into a multidomain state by the magnet's own magnetostatic field. For this reason the quest for improved energy product has involved a search for compounds with a large magnetization combined with the strong uniaxial anisotropy needed to develop hysteresis.

In former years, the problem was to achieve the necessary anisotropy, but more recently the focus has shifted to the problem of enhancing the magnetization. Modern highperformance magnets such as $\mathrm{SmCo}_{5}, \mathrm{Nd}_{2} \mathrm{Fe}_{14} \mathrm{~B}$, or the interstitial nitride $\mathrm{Sm}_{2} \mathrm{Fe}_{17} \mathrm{~N}_{3}$ consist of $3 d$ atoms which are exchange coupled to rare-earth atoms which provide the uniaxial anisotropy required of a permanent magnet. ${ }^{1-7}$ In reality, coercivity as high as $4.4 \mathrm{~T}$ has been achieved in $\mathrm{Sm}_{3} \mathrm{Fe}_{17} \mathrm{~N}_{3}$-based magnets, ${ }^{8}$ hut for practical purposes there is usually no call for coercivity very much greater than $M_{0} / 2$. On the other hand, the magnetization is reduced due to the rare-earth and nonmagnetic elements. The light rareearths' atomic moments are at best slightly larger than that of iron, but they occupy more than three times the volume. Nevertheless, it has been possible to use $\mathrm{Nd}_{2} \mathrm{Fe}_{14} \mathrm{~B}$ which has $\mu_{0} M_{0}=1.61 \mathrm{~T}$ and $\mu_{0} M_{0}^{2} / 4=516 \mathrm{~kJ} / \mathrm{m}^{3}$ to achieve roomtemperature energy products exceeding $400 \mathrm{~kJ} / \mathrm{m}^{3}$ in laboratory-scale magnets. ${ }^{2}$ At about $200{ }^{\circ} \mathrm{C}$, which is a technologically important temperature region, $\mathrm{Sm}_{2} \mathrm{Fe}_{17} \mathrm{~N}_{3}$ is potentially the best permanent magnetic material, ${ }^{1,5}$ but here suitable processing methods have not yet been developed.

Energy product has doubled every twelve years since the beginning of the century, and much effort is being made to yield further quantitative and qualitative progress in the per- formance of permanent magnetic materials. ${ }^{9}$ However, the outlook for discovering new ternary phases with magnetization significantly higher than that of those available, at present, is poor. ${ }^{3}$ Permanent magnets based on the appearance of a new rare-earth iron intermetallic phase could offer better temperature stability, yet higher anisotropy field or improved corrosion resistance, but the scope for significant improvements in the spontaneous magnetization is very limited. For instance, interstitial modification with small atoms such as nitrogen or carbon is effective for enhancing Curie temperature and anisotropy, ${ }^{1,5,10,11}$ but it has rather little effect on the magnetization- - the moment increase is, on the whole, a zero sum game. ${ }^{3}$

In the case of isotropic magnets, which are often more easy to produce than oriented magnets, the comparatively low remanence $M_{r} \approx M_{0} / 2$ reduces the theoretical energy product by a factor 4 . However, the production of nanocrystalline composites such as $\mathrm{Nd}_{2} \mathrm{Fe}_{14} \mathrm{~B} / \mathrm{Fe}_{3} \mathrm{BFe}$ and $\mathrm{Sm}_{2} \mathrm{Fe}_{17} \mathrm{~N}_{3} / \mathrm{Fe}$ by melt spinning ${ }^{12}$ and mechanical alloying, ${ }^{13}$ respectively, shows that it is possible to combine the high magnetization of soft-magnetic materials and the surplus anisotropy of rare-earth intermetallics. Soft magnetic phases such as bcc iron often reduce the energy product by degrading coercivity, but if the soft regions are small enough then exchange coupling stabilizes the magnetization direction of the soft phase. This kind of exchange coupling improves the low remanence of the isotropic hard phase, ${ }^{12-16}$ but the energy product, though improved with respect to the isotropic single-phase rare-earth material, does not reach the level attained in oriented single-phase rare-earth magnets. In other words, new approaches are necessary if the energy product is ever to doúble again.

Recent work by Skomski and Coey ${ }^{3,17,18}$ has shown how it should be possible to substantially increase the energy product in oriented nanostructured two-phase magnets by exploiting exchange coupling between hard and soft regions (Fig. 1). The idea behind these systems is to break out of the straightjacket of natural crystal structures by artificially structuring new materials. The concept is similar to that of the $4 f-3 d$ intermetallics themselves, but on a different scale, where the atoms are replaced by a mesoscopically structured hard-magnetic skeleton with surplus anisotropy and small soft-magnetic blocks. Based on analytical calculations, a 


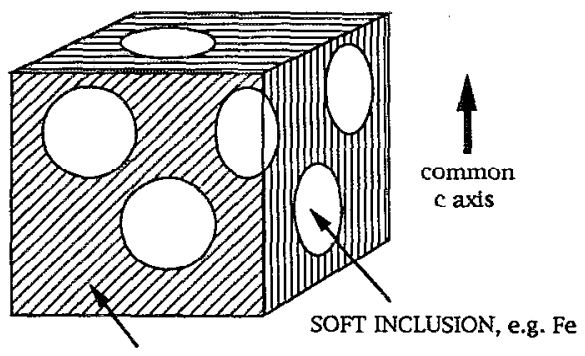

HARD MATRIX, e.g. 2-17 NITRIDE

FIG. 1. Spherical soft inclusions in an aligned hard matrix.

well-defined and realistic upper limit to the energy product of permanent magnets has been established, and energy products of order $1 \mathrm{MJ} / \mathrm{m}^{3}$ have been predicted for suitable multilayered and random structures.

Here, we summarize the background and results of these calculations, relate them to the problem of remanence enhancement in isotropic magnets, and draw conclusions with respect to future developments in permanent magnetism.

\section{BACKGROUND}

\section{A. Micromagnetic free energy}

To predict the performance of a permanent magnetic material we have to calculate the average magnetization $\langle\mathbf{M}\rangle$ as a function of the applied magnetic field $\mathbf{H}=H \mathbf{e}_{z}$. A convenient starting point is the (magnetic) free energy $F$, where the properties of the magnetic material enter as temperaturedependent parameters. Locally stable magnetic configurations are obtained by putting $\delta F / \delta \mathbf{M}(\mathbf{r})=0$, where $\delta . . . / \delta \mathbf{M}(\mathbf{r})$ denotes the functional derivative with respect to the magnetization $M(\mathbf{r})=M_{0}(\mathbf{r}) \mathbf{s}(\mathbf{r})$ with $s^{2}=1$. Typically, the free energy of permanent magnets exhibits two or more metastable equilibrium states, and tracing the magnetic configuration $\mathbf{s}(\mathbf{r})$ as a function of an external field $\mathbf{H}=H \mathbf{e}_{z}$ is quite a demanding task.

On a mesoscopic level, i.e., assuming that the magnetization is a continuous variable, the free energy of a permanent magnet reads ${ }^{19,20}$

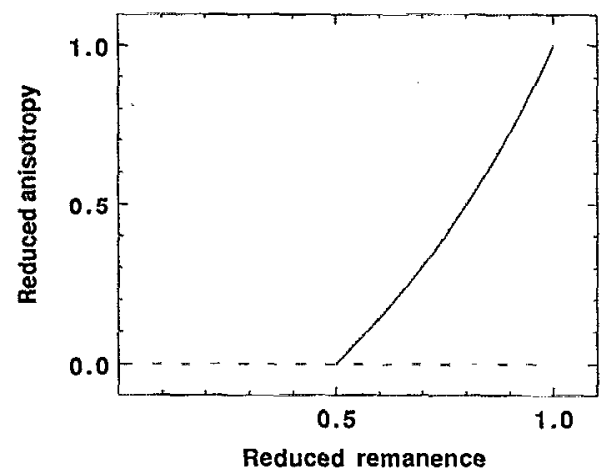

FIG. 2. Dependence of the reduced effective anisotropy constant $K_{\text {eff }} / K_{h}$ on the reduced remanence $M^{\prime} / M_{h} \cdot M^{\prime} / M_{h}=1 / 2$ and $M_{r} / M_{h} \rightarrow 1$ mean isotropic texture and complete alignment, respectively.

$$
F=\int\left[\eta_{x c}(\mathbf{r})+\eta_{a}(\mathbf{r})+\eta_{H}(\mathbf{r})+\eta_{m s}(\mathbf{r})\right] d \mathbf{r} .
$$

The contributions to the free-energy density are (i) the exchange frec-energy density $\eta_{x c}=A(\mathbf{r})[\nabla \mathrm{s}(\mathbf{r})]^{2}$, where the exchange stiffness $A(\mathbf{r})$ describes the tendency towards parallel spin alignment, (ii) the energy density of the (uniaxial) anisotropy

$$
\begin{aligned}
\eta_{a}= & -\left(K_{1}+2 K_{2}+3 K_{3}\right)(\mathbf{n s})^{2}+\left(K_{2}+3 K_{3}\right)(\mathbf{n s})^{4} \\
& -K_{3}(\mathbf{n s})^{6},
\end{aligned}
$$

where $\mathbf{n}$ is the unit vector of the local $c$-axis direction, (iii) the Zeeman energy density $\eta_{H}=-\mu_{0} M_{0}(\mathbf{r}) H s_{z}(\mathbf{r})$, where $\mathbf{H}=H \mathbf{e}_{z}$ is the external magnetic field, and (iv) the magnetostatic dipole interaction

$$
\begin{aligned}
\int \eta_{m s}(\mathbf{r}) d \mathbf{r}= & -\frac{1}{2} \sum_{i, j=1}^{3} \int \mathbb{K}_{i j}\left(\mathbf{r}-\mathbf{r}^{\prime}\right) \\
& \times M_{i}(\mathbf{r}) M_{j}\left(\mathbf{r}^{\prime}\right) d \mathbf{r} d \mathbf{r}^{\prime},
\end{aligned}
$$

with $\quad \mathbb{K}_{i j}(\mathbf{r})=\mu_{0}\left(3 r_{i} r_{j}-\delta_{i j} r^{2}\right) /\left(4 \pi r^{5}\right) \quad$ and $\quad \mathbf{M}(\mathbf{r})$ $=\Sigma_{i} M_{i}(\mathbf{r}) \mathbf{e}_{i}$. Note that putting $\mathbf{n}(\mathbf{r})=\mathbf{e}_{z}$ in Eq. (2) is equivalent to the familiar expression $\eta_{a}=K_{1} \sin ^{2} \theta$ $+K_{2} \sin ^{4} \theta+K_{3} \sin ^{6} \theta$, where $\theta$ is the angle between magnetization and $z$ direction.

\section{B. Coercivity and energy product}

Mechanical work to be done by a permanent magnet implies a magnetic hardness (coercivity) which keeps the magnetization in the desired direction. If the coercivity $H_{c}$ is too small, or if the hysteresis loop has an unfavorable shape, then the energy product won't reach the theoretical value $\mu_{0} M_{0}^{2} / 4$ deduced from the saturation magnetization $M_{0}$.

Depending on the real structure of a permanent magnet, there are different hardening mechanisms. Here we treat aligned nanostructured two-phase magnets as nucleationcontrolled magnets. The nucleation field $H_{N}=-H$ is defined as the (reverse) field at which the original state $\mathbf{s}(\mathbf{r}) \approx \mathbf{e}_{z}$ becomes unstable. Mathematically, this requires the determinant of the continuous matrix $\delta^{2} F / \delta \mathbf{s}(\mathbf{r}) \delta s\left(\mathbf{r}^{\prime}\right)$ to vanish. Note, however, that the introduction of pinning centers is a potential way of improving the coercivity by inhibiting the propagation of the reversed nucleus, so $H_{N} \leqslant H_{c}$ is a lower limit to the coercivity.

The determination of $H_{N}$ is a fairly demanding task, ${ }^{19-21}$ and we have to introduce suitable approximations. Due to the long-range character of $\mathbb{K}_{i j}(\mathbf{r})$, the magnetostatic interaction is the most complicated one. A simple, though nontrivial, approximation is to replace the external magnetic field by $H_{\text {eff }}=H-D\left\langle M_{z}\right\rangle$, where $D$ is the demagnetizing factor. It has been shown quite generally ${ }^{20-22}$ that for a homogeneous ellipsoid

$$
H_{c} \geqslant \frac{2 K_{1}}{\mu_{0} M_{0}}-D M_{0},
$$

where the factor $D M_{0}$ arises from the gain in magnetostatic energy due to an incoherent rotation process. The $>$ sign in Eq. (4) indicates that incoherent rotation costs exchange en- 
ergy, but the corresponding coercivity contribution scales as $A /\left(\mu_{0} M_{0} L^{2}\right)$, where $L$ is the magnet's size, and is practically negligible. In the case of coherent rotation (the StonerWohlfarth model $), H_{N}=2 K_{1} / \mu_{0} M_{0}-(1-3 D) M_{0} / 2$, and there is no influence of a "demagnetizing" field in spherical magnets where $D=1 / 3$. The result of the Stoner-Wohlfarth model is consistent with Eq. (4).

Equation (4) is called Brown's paradox, because real permanent magnets always seem to violate this inequality. This violation is due to the existence of inhomogenities in real magnets while Eq. (4) applies to homogeneous ellipsoids only. $^{21,22}$ In the following, we restrict ourselves to the socalled "intrinsic" $D=0$ hysteresis loop of macroscopic magnets, from which the energy product is determined. ${ }^{23}$

\section{MODEL AND CALCULATION}

To determine the nucleation field we assume ideal alignment of the hard phase, i.e., $\mathbf{n}(\mathbf{r})=\mathbf{e}_{z}$, and rewrite $\mathbf{s}(\mathbf{r}) \approx \mathbf{e}_{z}$ as

$$
\mathbf{s}=\sqrt{1-m^{2} \mathbf{e}_{z}}+\mathbf{m}, \quad \mathbf{m}=s_{x} \mathbf{e}_{x}+s_{y} \mathbf{e}_{y}, \quad m^{2} \ll 1 .
$$

Now series expansion of Eq. (1) yields the quadratic form

$$
F=\int\left[A(\mathbf{r})\{\nabla \mathbf{m}\}^{2}+K_{1}(\mathbf{r}) \mathbf{m}^{2}+\frac{1}{2} \mu_{0} M_{0}(\mathbf{r}) H \mathbf{m}^{2}\right] d \mathbf{r} .
$$

Recall that $K_{2}$ and $K_{3}$ do not enter this expression, ${ }^{19}$ so the nucleation field of ideally anisotropic hard magnets depends on $K_{1}$ only. The case of partially anisotropic and isotropic magnets will be discussed in Sec. IV. The equation of state $\delta F / \delta \mathbf{m}(\mathbf{r})=0$ is given by the identity

$$
\frac{\delta F}{\delta \mathbf{m}(\mathbf{r})}=-\nabla\left(\frac{\partial \eta}{\partial \nabla \mathbf{m}(\mathbf{r})}\right)+\frac{\partial \eta}{\partial \mathbf{m}(\mathbf{r})}
$$

and reads

$$
A(\mathbf{r}) \nabla^{2} \mathbf{m}+\nabla A(\mathbf{r}) \nabla \mathbf{m}-\left[2 K_{1}(\mathbf{r})+\mu_{0} M_{0}(\mathbf{r}) H\right] \mathbf{m}=0 .
$$

Except for the $\nabla A(\mathbf{r}) \nabla \mathbf{m}$ term (see Sec. IV), Eq. (8) is equivalent to Schrödinger's equation for a particle moving in a three-dimensional potential $2 K_{1}(\mathbf{r})+\mu_{0} M_{0}(\mathbf{r}) H$, which allows us to apply ideas familiar from quantum mechanics to discuss micromagnetics. Note that the $x$ and $y$ components of $\mathbf{m}=m_{x} \mathbf{e}_{\boldsymbol{x}}+m_{y} \mathbf{e}_{\boldsymbol{y}}$ are decoupled and degenerate in Eq. (8), so the vector $\mathbf{m}(\mathbf{r})$ can be replaced by any unspecified nucleation mode $\Psi(\mathbf{r})$. It is, however, convenient to think of $\Psi(\mathbf{r})$ as the magnetization $m_{x}(\mathbf{r})$ in $x$ direction.

Finally, for our inhomogeneous two-phase magnets we assume that the $\mathbf{r}$ dependence of the parameters $A, K_{1}$, and $M_{0}$ is given by $A_{h}, K_{h}, M_{h}$, and $A_{s}, K_{s}, M_{s}$ for hard (index $h$ ) and soft (index $s$ ) regions, respectively. Let the volume fraction of the hard regions become $f_{h}$; the fraction of the soft regions is then given by $f_{s}=1-f_{h}$.

The nucleation field $H_{N}$ is obtained as lowest eigenvalue of the Eq. (8). In the homogeneous "constant-potential" case, the ground-state eigenfunction is a plane wave with $\mathbf{k}=0$, and Eq. (8) reduces to the familiar form $H_{N}=-H=2 K_{1} / \mu_{0} M_{0}$. In the case of inhomogeneous magnets, the nucleation problem Eq. (8) can be solved by series expansion or an appropriate ansatz, which has been done for special one-, two-, and three-dimensional configurations. ${ }^{17,19,24-27}$ An example is a spherical soft inclusion (diameter $D, K_{s}=0$ ) in a hard-magnetic matrix (cf. Fig. 1). Introducing spherical coordinates and using the interface boundary condition $A_{s}(\mathbf{e} \cdot \nabla) \Psi_{s}=A_{h}(\mathbf{e} \cdot \nabla) \Psi_{h}$ we obtain the eigenvalue equation ${ }^{17}$

$$
\begin{gathered}
\frac{A_{s}}{A_{h}}\left\{\frac{D}{2} \sqrt{\frac{\mu_{0} M_{s} H_{N}}{2 A_{s}}} \cot \left(\frac{D}{2} \sqrt{\frac{\mu_{0} M_{s} H_{N}}{2 A_{s}}}\right)-1\right\}+1 \\
+\frac{D}{2} \sqrt{\frac{2 K_{h}-\mu_{0} M_{h} H_{N}}{2 A_{h}}}=0,
\end{gathered}
$$

which can be solved numerically. It turns out that the nucleation field reaches a high-coercivity plateau if the size of the soft inclusion is smaller than the Bloch wall thickness $\delta_{h}=\pi\left(A_{h} / K_{h}\right)^{1 / 2}$ of the hard phase. This plateau corresponds to complete exchange coupling. If the diameter $D$ of the soft inclusion is too large, then the magnetization becomes unstable and the coercivity falls off as $1 / D^{2}$.

For sufficiently small reverse fields $|H|<H_{N}(D)$ the single soft inclusion is perfectly aligned and slightly enhances the remanence provided $M_{s}>M_{h}$. On the other hand, when the distance between the soft inclusions is small, the magnetization modes can "tunnel" through the hard region which no longer acts as an effective potential barrier. In fact, this micromagnetic "exchange interaction" can reduce the nucleation field considerably when the thickness of the hard region is less than $\delta_{h}$. To obtain physically reasonable nucleation fields, the complete $A(\mathbf{r}), K_{1}(\mathbf{r})$, and $M_{0}(\mathbf{r})$ profiles have to be taken into account.

\section{RESULTS}

\section{A. Coercivity}

\section{Plateau limit}

In the plateau region, where the soft regions are very small, the problem can be treated in perturbation theory. ${ }^{3,17,18}$ As in quantum mechanics, the lowest order eigenvalue correction is obtained by using the normalized unperturbed function $\Psi_{0}$. This yields the nucleation field

$$
H_{N}=2 \frac{f_{s} K_{s}+f_{h} K_{h}}{\mu_{0}\left(f_{s} M_{s}+f_{h} M_{h}\right)} .
$$

Note that this result is independent of the shape of the soft and hard regions so long as the hard regions remain aligned.

\section{Multilayer IIm/t}

It is comparatively easy to treat one-dimensional structures such as multilayers, ${ }^{27}$ since the micromagnetic multilayer problem is analogous to the periodic multiple quantum wcll problem. For a multilaycred structure of alternating soft and hard magnetic layers the nucleation field is given by the implicit equation ${ }^{17}$

$$
\begin{gathered}
\sqrt{\frac{2 K_{h}-\mu_{0} M_{h} H_{N}}{2 A_{h}}} \tanh \left(\frac{l_{h}}{2} \sqrt{\frac{2 K_{h}-\mu_{0} M_{h} H_{N}}{2 A_{h}}}\right) \\
=\frac{A_{s}}{A_{h}} \sqrt{\frac{\mu_{0} M_{s} H_{N}}{2 A_{s}}} \tan \left(\frac{l_{s}}{2} \sqrt{\frac{\mu_{0} M_{s} H_{N}}{2 A_{s}}}\right),
\end{gathered}
$$


where $l_{h}$ and $l_{s}$ denote the thicknesses of the hard and soft layers, respectively. Note, that this result does not depend on whether the orientation of the crystallographic $c$ axis of the hard phase is perpendicular or longitudinal.

\section{B. Energy product}

Equation (10) implies an ideally rectangular hysteresis loop with energy product $\mu_{0} M_{r}^{2} / 4$, remanence $M_{r}=f_{h}$ $M_{h}+f_{s} M_{s}$, and (minimum) coercivity $H_{N}=M_{r} / 2$. Putting $K_{s}=0$ in the plateau limit we obtain with $H_{N}=M_{r} / 2$ the maximum energy product

$$
(B H)_{\max }=\frac{1}{4} \mu_{0} M_{s}^{2}\left(1-\frac{\mu_{0}\left(M_{s}-M_{h}\right) M_{s}}{2 K_{h}}\right) .
$$

Due to the large $K_{h}$, the second term in the bracket is small so the energy product approaches the ultimate value of $\mu_{0} M_{s}^{2} / 4$. The corresponding volume fraction of the hard phase is $f_{h}=\mu_{0} M_{s}^{2} / 4 K_{h}$.

The intermetallic with the most favorable combination of magnetization and anisotropy is $\mathrm{Sm}_{2} \mathrm{Fe}_{17} \mathrm{~N}_{3}$. If we consider the $\mathrm{Sm}_{2} \mathrm{Fe}_{17} \mathrm{~N}_{3} / \mathrm{Fe}$ system and assume values $\mu_{0} M_{s}=2.15 \mathrm{~T}, \mu_{0} M_{h}=1.55 \mathrm{~T}$, and $K_{h}=12 \mathrm{MJ} / \mathrm{m}^{3}$, we obtain a theoretical energy product of $880 \mathrm{~kJ} / \mathrm{m}^{3}(110$ MGOe) for a volume fraction of only $7 \%$ of the hard phase. ${ }^{17}$ A further increase of the energy product is possible if iron is replaced by $\mathrm{Fe}_{65} \mathrm{Co}_{35}$ with $\mu_{0} M_{s}=2.43 \mathrm{~T}$-the theoretical energy product of the $\mathrm{Sm}_{2} \mathrm{Fe}_{17} \mathrm{~N}_{3} / \mathrm{Fe}_{65} \mathrm{Co}_{35}$ system might be as high as $1090 \mathrm{~kJ} / \mathrm{m}^{3}$ (137 MGOe), with $f_{h}=9 \%$. It is remarkable that these optimum magnets are almost entirely composed of $3 d$ metals, with only about 2 -wt $\%$ samarium.

Another possibility is a multilayered structure of alternating soft and hard magnetic layers. Assuming $A_{s}=1.67 \times 10^{-11} \mathrm{~J} / \mathrm{m}$ and $A_{h}=1.07 \times 10^{-11} \mathrm{~J} / \mathrm{m}$ we deduce from Eq. (11) that a $\mathrm{Sm}_{2} \mathrm{Fe}_{17} \mathrm{~N}_{3} / \mathrm{Fe}_{65} \mathrm{Co}_{35}$ "'megajoule magnet" is obtained for layer thicknesses $l_{h}=2.4 \mathrm{~nm}$ and $l_{s}=9.0 \mathrm{~nm}$. The macroscopic shape of the magnet must of course correspond to the optimum operating point on the $B H$ curve; it should approximate an ellipsoid with $c / a=0.55$. Note that $A$ is generally of order $10^{-11} \mathrm{~J} / \mathrm{m}$, so $H_{N}$ and $(B H)_{\max }$ do not depend very much on the exchange-stiffness inhomogenity.

\section{Texture}

As we have seen, $K_{2}(\mathbf{r})$ and $K_{3}(\mathbf{r})$ do not influence the nucleation field so long as the unit vector in the easy axis direction $\mathbf{n}$ remains parallel to the field $\mathbf{e}_{z}$. The situation becomes much more difficult when the hard regions are only partially aligned or even isotropic. For instance, the components $m_{x}$ and $m_{y}$ of $\mathbf{m}$ [cf. Eq. (8)] are now coupled in a very complicated way. However, in lowest order perturbation theory the $m_{x}$ and $m_{y}$ decouple, and after some calculation we find that $K_{1}$ in Eq. (8) has to be replaced by

$$
\begin{aligned}
K_{\mathrm{eff}}= & -\frac{1}{2}\left(K_{1}+2 K_{2}+4 K_{3}\right)+\frac{3}{2}\left(K_{1}+4 K_{2}+9 K_{3}\right)\left\langle n_{z}^{2}\right\rangle \\
& -\frac{5}{2}\left(2 K_{2}+9 K_{3}\right)\left\langle n_{z}^{4}\right\rangle+\frac{21}{2} K_{3}\left\langle n_{z}^{6}\right\rangle .
\end{aligned}
$$

The ensemble average is defined as $\langle\ldots\rangle=\Omega^{-1} \int \ldots f(\theta) \sin \theta d \theta$ where $f(\theta)$ denotes the texture function of the hard phase. Note that Eq. (13) reduces to $K_{\text {eff }}=K_{1}$ in the ideally anisotopic limit $n_{z}=1$.

To discuss the overall behavior of $K_{\text {eff }}$ we use the singleparameter texture function $f(\theta)=(1+\nu) \cos ^{\nu} \theta^{28}$ and restrict ourselves to the case $K_{2}=K_{3}=0$. Using $n_{z}=\cos \theta$ we obtain

$$
K_{\mathrm{eff}}=K_{h}\left(\frac{2 M^{\prime}-M_{h}}{2 M_{h}-M^{\prime}}\right),
$$

where $M^{\prime}$ is the remanence of the hard regions in the interaction-free limit, i.e., $M^{\prime}=M_{h}$ for ideally anisotropic magnets and $M^{\prime}=0.5 M_{h}$ for isotropic magnets. In lowest order perturbation theory the nucleation field is proportional to $K_{\text {eff }}$, so $H_{N}=0$ for $M^{\prime}=0.5 M_{h}$ in this approximation. This means, that the nucleation field vanishes in the isotropic limit if soft and hard regions are extremely small. An alternative interpretation is given by Eq. (12), where $K_{h}$ has to be replaced by $K_{\text {eff }}$. The smallness of the $1 / K_{h}$ correction term in Eq. (12) requires the effective anisotropy to be very large, ${ }^{29}$. which is contradictory to $K_{\text {eff }}=0$, and it is not possible to apply Eq. (12) to isotropic materials.

\section{Isotropic magnets}

In the case of an isotropic matrix with randomly oriented hard regions alternative methods have to be used to calculate the coercivity. An estimate is obtained from a simple model where two hard regions with $\mathbf{n}_{1}=\mathbf{e}_{z}$ and $\mathbf{n}_{2}= \pm \mathbf{e}_{x}$ arc subject to an effective coupling $A / R_{\mathrm{eff}}^{2}$. As it can be shown easily, the weak-coupling limit (isolated grains) yields $M_{r}=M_{h} / 2$. In the case of strong exchange coupling the orientation of the grains is given by $\mathbf{s}_{1} \approx \mathbf{s}_{2} \approx \mathbf{e}_{x} / \sqrt{2}+\mathbf{e}_{z} / \sqrt{2}$, which yields the enhanced remanence $M_{r}=M_{h} / \sqrt{2}$. Note that the instability of the aligned $\mathbf{M}(\mathbf{r})=M_{h} \mathbf{e}_{z}$ state implies $M_{r}<M_{h}$ for remanence-enhanced single-phase magnets.

On the other hand, exchange coupling destroys coercivity. For weak coupling we find $H_{c}=2 K_{h} / \mu_{0} M_{h}$, while the strong-coupling coercivity $H_{c}=K_{1}^{2} R_{\text {eff }}^{2}\left(\sqrt{2} \mu_{0} M_{h} A\right)^{-1}$ decreases with increasing exchange interaction. As expected, this result reduces to $H_{c}=0$ in the "plateau limit" (infinitely large $A$ or infinitely small $\left.R_{\text {eff }}\right)$.

\section{DISCUSSION}

\section{A. Performance and processing}

A conceivable way to exploit the surplus anisotropy in modern rare-earth permanent magnets is to use aligned twophase magnets where the hard phase acts as a skeleton which keeps the magnetization of the soft phase in the desired direction. Micromagnetic analysis shows that the nucleation field in suitable nanostructured materials is proportional to the volume-averaged anisotropy.

Equation (12), which represents a hardness expansion with respect to the small parameter $\mu_{0}\left(M_{s}-M_{h}\right) M_{s} / K_{1}$, predicts the energy product of aligned nanostructured twophase magnets to be nearly as high as the ultimate value $\mu_{0} M_{0}^{2} / 4$. From the point of view of exchange coupling, the 
mechanism is similar to the remanence enhancement in isotropic magnets, but yields a saturation magnetization which is larger than that of the aligned hard phase.

The practical problem is to realize a structure where the soft regions are sufficiently small to avoid nucleation at small fields while having the hard regions crystallographically oriented. One conceivable solution is a disordered twophase magnet with a common $c$ axis throughout the hard regions. The energy product Eq. (12) is independent of the shape of the soft regions, so long as their size lies in the plateau region. However, soft regions much larger than this plateau size drastically reduce the nucleation field, ${ }^{29}$ and it is difficult to see how the formation of large soft-magnetic clusters might be avoided in practice. Furthermore, the hard skeleton has to be aligned to avoid the anisotropy reduction described by Eq. (14). Note that remanence-enhanced isotropic two-phase magnets operate on a slightly larger length scale.

A more realistic possibility may be a multilayered structure of alternating soft- and hard-magnetic layers. Conceivable production methods are laser ablation deposition ${ }^{30}$ or molecular-beam epitaxy. As discussed in Sec. IV, the permanent-magnetic performance of the multilayer is independent of whether the hard phase is perpendicularly or longitudinally aligned. It is worthwhile mentioning that permanent magnetic multilayers are one-dimensional systems, whose cigenmodes are subject to weak localization (Anderson localization). Weak localization in one-dimensional systems is caused by an arbitrarily small disorder, ${ }^{31,32}$ and second-order perturbation theory ${ }^{29}$ yields zero coercivity if the disorder is described by Gaussian or Poisson correlations. The reason for this coercivity breakdown is the small but finite probability of thick soft layers, since the coercivity is determined by the thickest soft layer around which the nucleation mode is localized. This means that weak localization does not destroy coercivity if there is an upper limit to the thickness of the soft layers.

Equation (1) is based on classical micromagnetic considerations; the sizes of the hard and soft regions must be large compared to atomic dimensions so that a continuum model can be applied. The model must break down when $l_{s}$ or $l_{h}$ is smaller than about $1 \mathrm{~nm}$. However, due to the small prefactor $\left(M_{s}-M_{h}\right) / M_{s}, 0.28$ for the $\mathrm{Sm}_{2} \mathrm{Fe}_{17} \mathrm{~N}_{3} / \mathrm{Fe}$ system, the energy product is not much affected if the fraction of the hard phase is increased. We still have an energy product of almost $800 \mathrm{~kJ} / \mathrm{m}^{3}$ (100 MGOe) in the $\mathrm{Sm}_{2} \mathrm{Fe}_{17} \mathrm{~N}_{3} / \mathrm{Fe}$ system when $f_{h} \approx 30 \%$, and we can use the extra hard material to outweigh quantum-mechanical size effects, to improve the thermal stability, suppress the effect of random stray fields and to create pinning centers. ${ }^{17,29}$

\section{B. Outlook}

Table I gives a tentative discussion of the question to what extent "giant-energy product" magnets will ever be exploited commercially. At present, the actual production of these structures represents a realistic but nevertheless extremely demanding challenge. In other words, the advantages of hypothetical "megajoule" magnets-very high energy product and low raw material costs-are largely outweighed
TABLE I. Permanent magnet processing and applications.

\begin{tabular}{llll}
\hline Material & Performance & $\begin{array}{c}\text { Processing } \\
\text { costs }\end{array}$ & $\begin{array}{c}\text { Raw material } \\
\text { price }\end{array}$ \\
\hline Steel & low & low & low \\
Ferrites & moderate & moderate & low \\
$\mathrm{SmCO}_{5}$ & high & moderate & high \\
$\mathrm{Nd}_{2} \mathrm{Fe}_{14} \mathrm{~B}$ & high & moderate & moderate \\
$\mathrm{Sm}_{2} \mathrm{Fe}_{17} \mathrm{~N}_{3}$ & high & high & moderate \\
$" \mathrm{MJ}^{\prime}$ magnets & very high & very high & low \\
\hline
\end{tabular}

by the complicated processing requirements. We therefore believe that the use of aligned nanostructured two phase magnets will be restricted to special applications such as micromechanics or thin-film electronics.

Of course, this emphasis on processing is likely to be a feature of all future permanent magnets, even if we include "exotic" systems such as magnetically hard roomtemperature superconductive currents or nanoscale magnetic clusters where the $>$ sign in Eq. (4) can be utilized. ${ }^{9}$ Let us imagine, for example, that it is possible to produce a singlephase permanent magnet somewhat better than $\mathrm{Nd}_{2} \mathrm{Fc}_{14} \mathrm{~B}$ by quenching from a high-pressure equilibrium state. The processing of this material will almost certainly be much more expensive than that of $\mathrm{Nd}_{2} \mathrm{Fe}_{14} \mathrm{~B}$, leading to much less widespread commercial applications. In our opinion, singular events such as the discovery of $\mathrm{Nd}_{2} \mathrm{Fe}_{14} \mathrm{~B}$ are unlikely to happen again, and most future technological and scientific progress will be in directions other than improving the energy product.

\section{CONCLUSIONS}

In conclusion, it is likely that substantial improvements in the energy product of permanent magnets can be achieved by exchange hardening in nanoscale combinations of a soft phase and an oriented hard phase, structured according to the principles we have outlined. For example, the maximum energy product of nanostructured $\mathrm{Sm}_{2} \mathrm{Fe}_{17} \mathrm{~N}_{3} / \mathrm{Fe}_{65} \mathrm{Co}_{35}$ multilayers is predicted to be as high as $1090 \mathrm{~kJ} / \mathrm{m}^{3}$ (137 MGOe). The high energy product, which is based on nucleationcontrolled coercivity, breaks down in the limit of remanenceenhanced isotropic magnets. The actual production of the new material-a demanding but realistic aim-will be expensive, which restricts the potential use of 'giant-energyproduct' magnets to special applications.

\section{ACKNOWLEDGMENTS}

The author is indebted to J. M. D. Coey for stimulating discussions and help in details. This work was supported by the BRITE/EURAM program of the European Commission. It forms part of the Concerted European Action on Magnets.

${ }^{1}$ J. M. D. Coey, Physica Scripta T39, 21 (1991).

${ }^{2}$ M. Sagawa, S. Hirosawa, H. Yamamoto, S. Fujimura, and Y. Matsuura, Jpn. J. Appl. Phys. 26, 785 (1987).

${ }^{3}$ J. M. D. Coey and R. Skomski, Physica Scripta T49, 315 (1993).

${ }^{4}$ M. Sagawa, S. Fujimura, N. Togawa, H. Yamamoto, and Y. Matsuira, J. Appl. Phys. 55, 2083 (1984).

${ }^{5}$ J. M. D. Coey and H. Sun, J. Magn. Magn. Mater. 87, L251 (1990). 
${ }^{6}$ G. J. Long and F. Grandjean, Supermagnets. Hard Magnetic Materials (Kluwer, Dordrecht, 1991).

${ }^{7}$ J. J. Croat, J. F. Herbst, R. W. Lee, and F. E. Pinkerton, J. Appl. Phys. 55, 2078 (1984).

${ }^{8}$ C. Kuhrt, K. O'Donnell, M. Katter, J. Wecker, K. Schnitzke, and L. Schultz, Appl. Phys. Lett. 60, 3316 (1992).

${ }^{9}$ M. Sagawa and J. M. D. Coey, Europe-Japan Workshop on the Future of Permanent Magnetism, Connemara, Ireland, March 13-16, 1994 (to be published).

${ }^{10}$ Q.-N. Qi, H. Sun, R. Skomski, and J. M. D. Coey, Phys. Rev. 45, 12278 (1992).

${ }^{11}$ R. Skomski, M. D. Kuz'min, and J. M. D. Coey, J. Appl. Phys. 73, 6934 (1993).

${ }^{12}$ R. Coehoorn, D. B. de Mooij, J. P. W. B. Duchateau, and K. H. J. Buschow, J. Phys. 49, C8-669 (1988).

${ }^{13}$ J. Ding, P. G. McCormick, and R. Street, J. Magn. Magn. Mater. 124, L1 (1993).

${ }^{14}$ K.-H. Müller, J. Schneider, A. Handstein, D. Eckert, P. Nothnagel, and H. R. Kirchmayr, Mater. Sci. Eng. A 133, 151 (1991).

${ }^{15} \mathrm{E}$. Kneller and R. Hawig, IEEE Trans. Magn. 27, 3588 (1991).

${ }^{16}$ E. Callen, Y. J. Liu, and J. R. Cullen, Phys. Rev. B 16, 263 (1977).
${ }^{17}$ R. Skomski and J. M. D. Coey, Phys. Rev. B 48, 15812 (1993).

${ }^{18}$ R. Skomski and J. M. D. Coey, IEEE Trans. Magn. 29, 2860 (1993).

${ }^{19} \mathrm{H}$. Kronmüller, Phys. Status Solidi B 144, 385 (1987).

${ }^{20}$ W. F. Brown, Magnetostatic Principles in Ferromagnetism (Interscience, New York, 1962).

${ }^{21}$ A. Aharoni, Rev. Mod. Phys. 34, 227 (1962).

${ }^{22}$ W. F. Brown, Rev. Mod. Phys. 17, 15 (1945)

${ }^{23}$ Of course, the practical realization of $(B H)_{\max }$ requires a shape which corresponds to the optimum operating point on the $B H$ curve.

${ }^{24}$ R. Skomski, Phys. Status Solidi B 174, K77 (1992).

${ }^{25} \mathrm{~J}$.-F. Hu and H. Kronmüller, Phys. Status Solidi B 172, 67 (1992).

${ }^{26}$ R. Skomski, K.-H. Müller, P. A. P. Wendhausen, and J. M. D. Coey, J. Appl. Phys. 73, 6047 (1993).

${ }^{27} \mathrm{~S}$. Nieber and H. Kronmüller, Phys. Status Solidi B 153, 367 (1989).

${ }^{28}$ L. Jahn, K. Elk, and R. Schumann, J. Magn. Magn. Mater. 104-107, 1439 (1992).

${ }^{29}$ R. Skomski and J. M. D. Coey, IEEE Trans. Magn. 30, 607 (1994).

${ }^{30}$ M. Enrech, R. Skomski, J. M. D. Coey, and J. G. Lunney, J. Appl. Phys. 73, 6421 (1993).

${ }^{31}$ P. W. Anderson, Phys. Rev. 109, 1492 (1958).

${ }^{32}$ P. A. Lee and T. V. Ramakrishnan, Rev. Mod. Phys. 57, 287 (1985). 